\title{
Modeling and Numerical Simulation of the Turbulent Two-Phase Jet Confined in the Cylindrical Channel
}

\author{
IVAN V. KAZACHKOV \\ Department of Information Technology and Data Analysis \\ Nizhyn Mykola Gogol State University \\ 16600 Grafska str. 2, Nizhyn \\ UKRAINE \\ kazachkov.iv@ndu.edu.ua, ivan.kazachkov@energy.kth.se
}

\begin{abstract}
Mixing processes in the turbulent two-phase jet confined at some distance from the nozzle are modeled and examined. Many natural and technical phenomena deal with the turbulent mixing and heat transfer in the jet of mutually immiscible liquids, which represent an important class of the modern multiphase systems dynamics. The differential equations for axially symmetrical two-dimensional stationary flow and the integral correlations in a cylindrical coordinate system are considered for the free heterogeneous jet confined at its initial or ground part in the cylindrical channel. Algorithm and the results obtained may be of interest for the research and industrial tasks, where the calculations of the turbulent mixing and heat transfer in multiphase jet devices are of importance.
\end{abstract}

Key-Words: - Confined Turbulent Jet; Cylindrical Channel; Two-Phase; Modeling; Computational Experiment Received: March 11, 2020. Revised: September 22, 2020. Accepted: October 1, 2020. Published: October 9 , 2020.

\section{Development of the Mathematical Model for Confined Two-Phase Jet}

\subsection{Introduction to the Model of Turbulent Two-Phase Confined Jet}

The free jet can be confined at its initial or ground part like it is done in a number of the jet devices for different thermal hydraulic applications, depending on the physical situation [1-9].

Let us start description of the physical situation and mathematical model developed with the general view of the turbulent two-phase jet flow shown in Fig. 1 [1]:

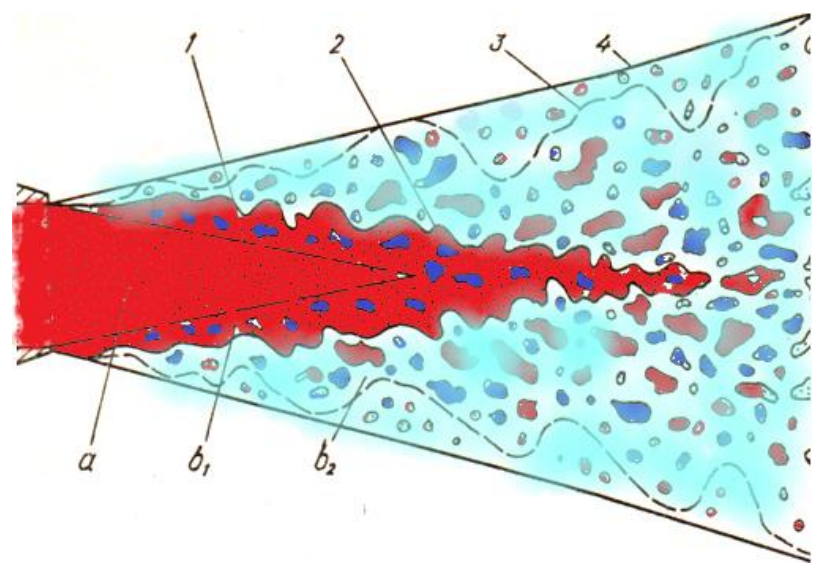

Fig. 1 General view of the multiphase turbulent jet in the pool of other immiscible liquid

and schematic representation for the two-phase turbulent jet confined by cylindrical channel at the distance $x_{g}$ on its initial part (Fig. 2) or its ground part (Fig. 3) [5]:

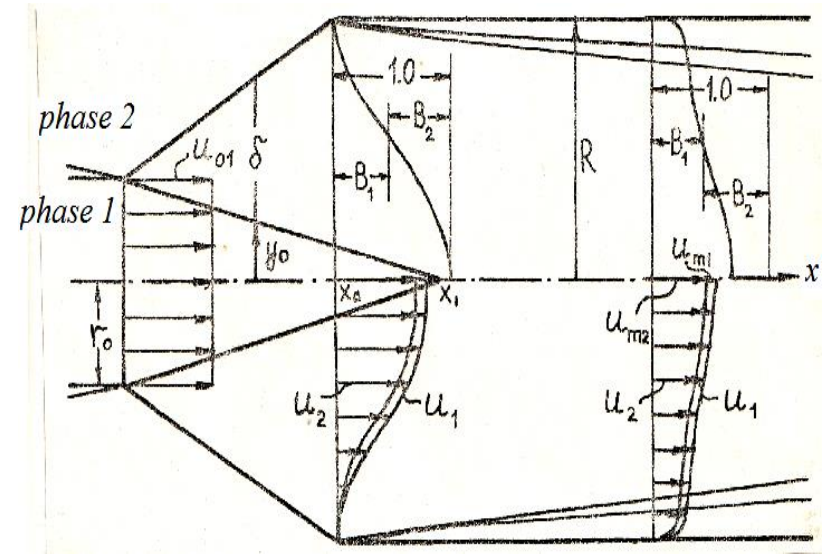

Fig. 2 Turbulent two-phase jet confined in cylindrical channel on its initial part

The structural scheme for the mixing process in Figs 2,3 is simplified: the initial part of the length $x_{\mathrm{i}}$ with the approximately linear boundaries for the conical surface (in cylindrical coordinate system) of the internal core of a first phase and mixing zone between internal and external boundaries of the jet. The turbulent zone contains fragments of the phases as far as immiscible liquids have behaviors like the separate phases, with their interfacial multiple surfaces. The first phase in a potential core is totally 
spent in an initial part of the mixing zone. Then a short transit area follows. Afterwards the ground part of the two-phase jet begins, with the two phases well mixed across the entire layer of a jet.

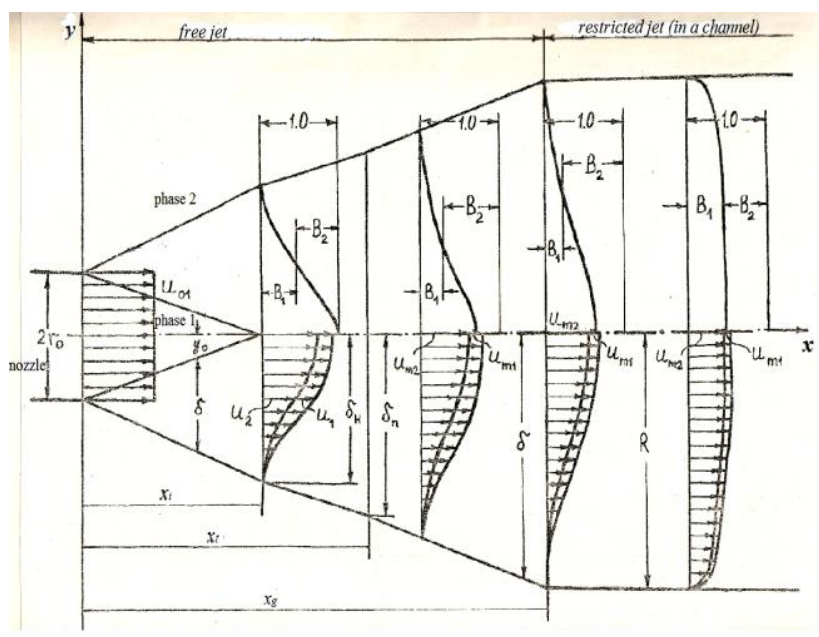

Fig. 3 Turbulent two-phase jet confined in cylindrical channel on its ground part, at the distance $x=x_{g}[1,5]$

The external boundary of a mixing zone in a free jet $[1-3,6,7]$, before its confining by cylindrical channel, is determined by zero longitudinal velocity of the second phase and zero transversal velocity of the first phase (the second phase is sucked from an immovable surrounding into a mixing zone). The function-indicator of the first phase $B_{1}(t)$ is zero at the external interface because it is absent in surrounding medium. Similar, the function-indicator $B_{2}(t)$ is zero on the boundary of the potential core, the interface of the first phase flowing from the nozzle. In a first approach, an influence of the mass, viscous and capillary forces are neglected.

Modeling and numerical simulation for the free turbulent two-phase flow was considered in $[1,2]$, for the confined jets - in $[4,5]$. The equation array for two-phase flow is used according to the method of Prof. Nakorchevskii [1] for multiphase flows based on introduction of the function-indicator.

The function-indicator $B_{i}(t)$ is introduced for the phases in multiphase flow by the next rule: $B_{i}(t)= \begin{cases}1, & i \text {-phase occupies elementary volume } \delta \mathrm{V} \\ 0, & i \text {-phase outside elementary volume } \delta \mathrm{V}\end{cases}$

all parameters $a^{l}(t)$ (density of liquid, flow velocity, temperature, etc.) of a mixture in the turbulent multiphase flow are considered as follows:

$$
a^{l}(t)=\sum_{i=1}^{m} B_{i}(t) a_{i}^{l}(t), \sum_{i=1}^{m} B_{i}=1 .
$$

The analog of the Navier-Stokes equations in a boundary layer approach is done in the form [1]:

$$
\begin{gathered}
\sum_{i=1}^{m}\left\lfloor\frac{\partial}{\partial x}\left(y \rho_{i} B_{i} u_{i}\right)+\frac{\partial}{\partial y}\left(y \rho_{i} B_{i} v_{i}\right)\right\rfloor=0, \\
\sum_{i=1}^{m} \rho_{i} B_{i}\left(u_{i} \frac{\partial u_{i}}{\partial x}+v_{i} \frac{\partial u_{i}}{\partial y}\right)=-\frac{d p}{d x}+\frac{1}{y} \frac{\partial}{\partial y}\left[y \sum_{i=1}^{m} B_{i} \tau_{i}\right]_{m},
\end{gathered}
$$

where the stationary equations (1) for the flow of incompressible liquids are written in a cylindrical coordinate system. Here are: $p$ - pressure, $\rho$ - density, $u, v$ - the longitudinal and transversal velocity components by $x$ and $y$, respectively, $\tau_{i}$ - turbulent stress in an $i$-phase.

The mathematical modeling and computer numerical simulation for the free two-phase jet were considered in $[6,7]$. This paper is continuing them for the case of the jet confined by cylindrical channel.

\subsection{Mathematical Model for the Turbulent Two-Phase Confined Jet}

\subsubsection{Basic Equations for the Confined Jet Flow}

For the jet confined at its initial part (Fig. 2), in its potential core, the Bernoulli equation is satisfied

$$
p+0.5 \rho_{1} u_{c 1}^{2}=p_{0}+0.5 \rho_{1} u_{01}^{2},
$$

where $u_{\mathrm{ci}}$ is velocity of the $i$-th phase in a jet's core, $x_{\mathrm{c}}$ - initial cross-section of the confining channel, $p_{0}$ pressure in a free jet. From the (2) follows

$$
u_{c 1}=u_{01} \sqrt{1-\bar{p}}, \quad \bar{p}=2\left(p-p_{0}\right) /\left(\rho_{1} u_{01}^{2}\right) .
$$

Using the transversal velocities of the phases from the first two equations (1), with account of the zero transversal velocities at the axis due to symmetry of a flow and at the wall of a channel due to its impermeability, we can get

$$
\begin{gathered}
\rho_{1} u_{01} r_{0}^{2}=\rho_{1} u_{c 1} y_{0}^{2}+2 \int_{y_{0}}^{R} \rho_{1} B_{1} u_{1}\left(y_{0}+y\right) d y, \\
\int_{y_{0 c}}^{R_{c}} \rho_{2} B_{2 c} u_{2 c}\left(y_{0 c}+y\right) d y=\int_{y_{0}}^{R} \rho_{2} B_{2} u_{2}\left(y_{0}+y\right) d y \\
0.5 \frac{d}{d x}\left(\rho_{1} u_{c 1}^{2} y_{0}^{2}\right)+\frac{d}{d x} \sum_{i=1}^{2} \int_{y_{0}}^{R} \rho_{i} B_{i} u_{i}^{2}\left(y_{0}+y\right) d y= \\
=R \sum_{i=1}^{2} B_{w i} \tau_{w i}-0.5 R^{2} \frac{d p}{d x}
\end{gathered}
$$




$$
\begin{gathered}
\frac{d}{d x}\left[\rho_{1} u_{c 1}\left(u_{c 1}-u_{1}^{*}\right) y_{0}^{2}+2 \sum_{i=1}^{2} \int_{y_{0}}^{y^{*}} \rho_{i} B_{i} u_{i}^{2}\left(y_{0}+y\right) d y\right]+ \\
-2 \sum_{i=1}^{2} u_{i}^{*} \frac{d}{d x} \int_{y_{0}}^{y^{*}} \rho_{i} B_{i} u_{i}\left(y_{0}+y\right) d y= \\
=2\left(y_{0}+y^{*}\right) \sum_{i=1}^{2} B_{i}^{*} \tau_{i}^{*}-\left(y_{0}+y^{*}\right)^{2} \frac{d p}{d x}
\end{gathered}
$$

where $R(x)$ is the radius of the confining channel, equal to the radius of a free jet at the cross section $x=x_{\mathrm{c}}$. Indexes $c$ and $w$ belong to $x=x_{\mathrm{c}}$ and to the wall of a channel, respectively. Star assigns the values at $y=y^{*}<\delta$, e.g. $y^{*}=0.5 \delta$. The third integral correlation in (4) was derived with account of the following two equations got from the first two equations of (4):

$$
\begin{gathered}
\frac{d}{d x} \int_{y_{0}}^{R} \rho_{1} B_{1} u_{1}\left(y_{0}+y\right) d y=-\frac{1}{2} \frac{d}{d x} \rho_{1} u_{c 1} y_{0}^{2}, \\
\frac{d}{d x} \int_{y_{0}}^{R} \rho_{2} B_{2} u_{2}\left(y_{0}+y\right) d y=0 .
\end{gathered}
$$

The equation array (4) contains two equations of the mass conservation for the first and second phase and the integral correlations of momentum - by total cross-section and by part of it. Thus, two algebraic and two ordinary differential equations represent the model of the confined two-phase jet for $x \geq x_{c}$, where by $x \geq x_{i}, y_{0}=0$, and the function-indicator of the first phase is not constant at the axis of a jet (channel): $B_{\mathrm{ml}}=B_{\mathrm{ml}}(x)$.

If similarly to the free jet, an assumption is accepted about the correlation

$$
u_{2 c} / u_{1 c}=u_{m 2} / u_{m 1}=s_{0}=\text { const, }
$$

then the system (4) is reduced to the following dimensionless form:

$$
\begin{gathered}
\sqrt{1-\bar{p}}\left[\bar{y}_{0}^{2}+2 \bar{\delta} \int_{0}^{1} B_{1} \bar{u}_{1}\left(\bar{y}_{0}+\bar{\delta} \eta\right) d \eta\right]=1 \\
\sqrt{1-\bar{p}} \int_{0}^{1}\left(1-B_{1}\right) \bar{u}_{2}\left(\bar{y}_{0}+\bar{\delta} \eta\right) \bar{\delta} d \eta= \\
=\int_{0}^{1}\left(1-B_{1 c}\right) \bar{u}_{2 c}\left(\bar{y}_{0 c}+\bar{\delta}_{c} \eta\right) \bar{\delta} d \eta \\
\frac{d}{d \bar{x}} \sum_{i=1}^{2} i_{0}^{i-1}(1-\bar{p}) \int_{0}^{1} B_{i} \bar{u}_{i}^{2}\left(\bar{y}_{0}+\bar{\delta} \eta\right) \bar{\delta} d \eta+ \\
0.5 \frac{d}{d \bar{x}} \bar{y}_{0}^{2}(1-\bar{p})=-\frac{\bar{R}^{2}}{4} \frac{d \bar{p}}{d \bar{x}}+\bar{R} \sum_{i=1}^{2} B_{i} \bar{\tau}_{i},
\end{gathered}
$$

$$
\begin{gathered}
\frac{d}{d \bar{x}} \sum_{i=1}^{2} i_{0}^{i-1}(1-\bar{p}) \int_{0}^{\eta^{*}} B_{i} \bar{u}_{i}^{2}\left(\bar{y}_{0}+\bar{\delta} \eta\right) \bar{\delta} d \eta+ \\
+0.5\left(1-\bar{u}_{1}^{*}\right) \frac{d}{d \bar{x}} \bar{y}_{0}^{2}(1-\bar{p})+ \\
-\sqrt{1-\bar{p}} \sum_{i=1}^{2} i_{0}^{i-1} \bar{u}_{i}^{*} \frac{d}{d \bar{x}} \sqrt{1-\bar{p}} \int_{0}^{\eta^{*}} B_{i} \bar{u}_{i}\left(\bar{y}_{0}+\bar{\delta} \eta\right) \bar{\delta} d \eta= \\
=\left(\bar{y}_{0}+\bar{\delta}^{*}\right) \sum_{i=1}^{2} B_{i}^{*} \bar{\tau}_{i}^{*}-0.25\left(\bar{y}_{0}+\bar{\delta} \eta^{*}\right)^{2} \frac{d \bar{p}}{d \bar{x}}
\end{gathered}
$$

where:

$$
\begin{aligned}
& \bar{\delta}=\frac{\delta}{r_{0}}, \eta=\frac{y-y_{0}}{\delta}, \bar{x}=\frac{x-x_{c}}{r_{0}}, \bar{y}_{0}=\frac{y_{0}}{r_{0}} \\
& \bar{R}=\frac{R}{r_{0}}, n=\frac{\rho_{2}}{\rho_{1}}, \bar{u}_{i}=\frac{u_{i}}{u_{c i} s^{i-1}}, \bar{\tau}_{i}=\frac{\tau_{i}}{\rho_{1} u_{01}^{2}}, i_{0}=n s_{0}^{2}
\end{aligned}
$$

The correlation (6) means that slip of the two phases is constant at least at the axis of the axially symmetrical flow, which may be rough for the big density ratio of liquid phases.

\subsubsection{The Boundary Conditions for Confined Jet}

The equations (4) satisfy the following boundary conditions

$$
x=x_{c}, \quad y_{0}=y_{0 c}, \quad p=p_{0}, \quad u_{i}=u_{i c}, \quad B_{1}=B_{l c} .
$$

At the $x>x_{i}, u_{i}=\bar{u}_{i} u_{m i}$ is. Then the boundary conditions are:

$$
\bar{x}=0, \bar{u}_{i}=\bar{u}_{i c}, B_{i}=B_{i c}, \bar{y}_{0}=\bar{y}_{0 c}, \bar{\delta}=\bar{\delta}_{c}, \bar{p}=0
$$

But at the initial confined part of a jet, when $x \in\left(x_{c}, x_{i}\right)$, there is also added

$$
\eta=0, \bar{u}_{i}=1, B_{m 1}=1 .
$$

\subsubsection{The Jet Confined at Its Initial Part}

After the end of the potential core (or in case a jet is confined at its initial part), $x>x_{i}$, the regularities of a jet's spreading are described by the system of mixed algebraic-differential equations:

$$
\begin{gathered}
2 \bar{R}^{2} \sqrt{1-\bar{p}} \int_{0}^{1} B_{1} \bar{u}_{1} \eta d \eta=1, \bar{\delta}=\bar{R}, \bar{y}_{0}=0, \\
\frac{d}{d \bar{x}}(1-\bar{p}) \bar{R}^{2} \sum_{i=1}^{2} i_{0}^{i-1} \int_{0}^{1} B_{i} \bar{u}_{i}^{2} \eta d \eta=\bar{R} \sum_{i=1}^{2} B_{w i} \bar{\tau}_{w i}-\frac{\bar{R}^{2}}{4} \frac{d \bar{p}}{d \bar{x}}, \\
\bar{R}^{2} \sqrt{1-\bar{p}} \int_{0}^{1}\left(1-B_{1}\right) \bar{u}_{2} \eta d \eta=
\end{gathered}
$$




$$
\begin{gathered}
=\int_{0}^{1}\left(1-B_{1 c}\right) \bar{u}_{2 c}\left(\bar{y}_{0 c}+\bar{\delta}_{c} \eta\right) \overline{\delta_{c}} d \eta \\
\frac{d}{d \bar{x}}(1-\bar{p}) \bar{R}^{2} \sum_{i=1}^{2} i_{0}^{i-1} \int_{0}^{\eta^{*}} B_{i} \bar{u}_{i}^{2} \eta d \eta= \\
=\sqrt{1-\bar{p}} \sum_{i=1}^{2} i_{0}^{i-1} \bar{u}_{i}^{*} \frac{d}{d \bar{x}} \bar{R}^{2} \sqrt{1-\bar{p}} \int_{0}^{\eta^{*}} B_{i} \bar{u}_{i} \eta d \eta+ \\
+\bar{R} \eta^{*} \sum_{i=1}^{2} B_{i}^{*} \bar{\tau}_{i}^{*}-\frac{1}{4} \bar{R}^{2}\left(\eta^{*}\right)^{2} \frac{d \bar{p}}{d \bar{x}} .
\end{gathered}
$$

\subsubsection{The Jet Confined at Its Ground Part}

If a jet is confined at its ground part, the basic equations are derived similarly to the above. In a dimension form:

$$
\begin{gathered}
\int_{0}^{R(x)} B_{i} u_{i} y d y=\int_{0}^{R_{c}} B_{i c} u_{i c} y d y, \quad(i=1,2), \\
\sum_{i=1}^{2} \rho_{i} B_{m i} \frac{d u_{m i}}{d x}=2\left(\frac{\partial}{\partial y} \sum_{i=1}^{2} B_{i} \tau_{i}\right)_{m}-\frac{d p}{d x}, \quad(12) \\
\frac{d}{d x} \sum_{i=1}^{2} \int_{0}^{y^{*}(x)} \rho_{i} B_{i} u_{i}^{2} y d y-\sum_{i=1}^{2} u_{i}^{*} \frac{d}{d x} \int_{0}^{y^{*}(x)} \rho_{i} B_{i} u_{i} y d y= \\
=y^{*} \sum_{i=1}^{2} B_{i}^{*} \tau_{i}^{*}-0.5\left(y^{*}\right)^{2} \frac{d p}{d x}, \\
\frac{d}{d x} \sum_{i=1}^{2} \int_{0}^{R(x)} \rho_{i} B_{i} u_{i}^{2} y d y=R(x) \sum_{i=1}^{2} B_{w i} \tau_{w i}-\frac{R^{2}(x)}{2} \frac{d p}{d x} .
\end{gathered}
$$

Further investigation of the stationary 2-D axially symmetrical heterogeneous two-phase turbulent jets in a confined domain is reasonable to conduct on some simplified models to reveal the basic features of such flows. The main problem with multiphase flows consists in closeness of the equation array.

The "new Prandtl formula" is used as before for the free jet flows. Peculiarity of the confined jet is an appearance of the boundary layer near the wall of a channel [8], which complicates the structure of a flow substantially. If a character of a jet flow is assumed inside a channel up to the wall, the both phases have slip on the wall. Then the structural scheme is considered similar to the proposed in [8] for the homogeneous jet, and $\eta^{*}$ is chosen the one corresponding to a developed jet flow.

Then the profiles of the parameters are

$$
\begin{aligned}
& u_{i}=\left(u_{m i}-u_{w i}\right) \bar{u}_{i c}+u_{w i}, \quad(i=1,2), \\
& B_{1}=\left(B_{m 1}-B_{w 1}\right) \bar{B}_{1}+B_{w 1}, \quad B_{2}=1-B_{1},
\end{aligned}
$$

where $\bar{u}_{1 c}, \bar{u}_{2 c}, \bar{B}_{1}$ are determined according to the above-considered method of two-phase flow.

The mathematical model developed allows considering the mutual interaction of the two-phase turbulent jet with the confining cylindrical channel. The main parameters are: $u_{m l}(x), u_{w l}(x), B_{m l}(x)$, $B_{w l}(x), p(x)$ and $R(x)$, one of which can be stated as the optimal in a desired way. For example, for the grading of a smooth stabilization channel, in case of jet's confining at its ground part, $\mathrm{d} p / \mathrm{d} x=0$ is put in (12). Then the functions $u_{m l}(x), u_{w l}(x), B_{m l}(x), B_{w l}(x)$ and $R(x)$ are determined according to the task stated.

For the stabilized flow, when $u_{m l}=u_{w l}=u_{1 \infty}, B_{m l}=$ $B_{w l}=B_{1 \infty}$, the mathematical model yields:

$$
\begin{gathered}
\bar{R}^{2} \bar{B}_{1 \infty} \bar{u}_{1 \infty}=2 \int_{0}^{1} \bar{B}_{1 c} \bar{u}_{1 c} \eta d \eta \\
\bar{R}^{2} \bar{B}_{1 \infty} \bar{u}_{1 \infty}=2 \int_{0}^{1} \bar{B}_{1 c} \bar{u}_{2 c} \eta d \eta+\frac{\bar{R}^{2} \bar{u}_{1 \infty}-2 \int_{0}^{1} \bar{u}_{2 c} \eta d \eta}{B_{m 1 c}}, \\
\frac{d \bar{p}}{d x}=-B_{m 1 c}\left[\bar{B}_{1 \infty}\left(1-i_{0}\right)+\frac{i_{0}}{B_{m 1 c}}\right] \frac{d \bar{u}_{1 \infty}^{2}}{d x} .
\end{gathered}
$$

From (2) follows that $\bar{R}^{2} \bar{u}_{1 \infty}$ independently on $\bar{R}, \bar{B}_{1 \infty}$ is determined by

$$
\begin{gathered}
C_{0}=\bar{R}^{2} \bar{u}_{1 \infty}=2 \int_{0}^{1} \bar{u}_{2 c} \eta d \eta+ \\
+2 B_{m 1 c}\left(\int_{0}^{1} \bar{B}_{1 c} \bar{u}_{1 c} \eta d \eta-\int_{0}^{1} \bar{B}_{1 c} \bar{u}_{2 c} \eta d \eta\right) .
\end{gathered}
$$

And the pressure correlation with a form of the confining channel is as follows

$$
\bar{p}+B_{m 1 c}\left[\bar{B}_{1 \infty}\left(1-i_{0}\right)+\frac{i_{0}}{B_{m 1 c}}\right] \frac{C_{0}^{2}}{\bar{R}^{4}}=\text { const }
$$

where $R=\bar{R} R_{c}, u_{1 \infty}=\bar{u}_{1 \infty} u_{m 1 c}$, index $\infty$ means a value of a parameter for the totally stabilized flow, $\tau_{w i}=0$ according to the accepted scheme.

\subsection{Equations for Jet Confined at Initial Part}

If a jet is confined at its initial part, with a simplification $h=h_{c}=$ const, it is described by the following equations.

For a jet's initial part $\left(B_{m l}=1, u_{m i}=u_{c 1} s_{0}^{i-1}\right)$ : 
$2 \sqrt{1-\bar{p}}\left(\bar{R}-\bar{y}_{0}\right) \int_{0}^{1}\left[\left(1-B_{w 1}\right) \bar{B}_{1 c}+B_{w 1}\right]\left[\bar{u}_{w 1}+\right.$

$\left.+\bar{u}_{1 c}\left(1-\bar{u}_{w 1}\right)\right]\left[\left(\bar{R}-\bar{y}_{0}\right) \eta+\bar{y}_{0}\right] d \eta=1-\bar{y}_{0}^{2} \sqrt{1-\bar{p}}$

$\sqrt{1-\bar{p}}\left(\bar{R}-\bar{y}_{0}\right) \int_{0}^{1}\left[\left(1-B_{w 1}\right)+\left(B_{w 1}-1\right) \bar{B}_{1 c}\right]$.

$\cdot\left[\left(1-\bar{u}_{w 1}\right) \bar{u}_{2 c}+\bar{u}_{w 1}\right]\left[\left(\bar{R}-\bar{y}_{0}\right) \eta+\bar{y}_{0}\right] d \eta=$

$=\left(\bar{R}_{c}-\bar{y}_{0 c}\right) \int_{0}^{1}\left(1-\bar{B}_{1 c}\right) \bar{u}_{2 c}\left[\left(\bar{R}_{c}-\bar{y}_{0 c}\right) \eta+\bar{y}_{0 c}\right] d \eta$,

$0.5 \bar{y}_{0}^{2}\left(\bar{R}-\bar{y}_{0}\right) \int_{0}^{1}\left[\left(1-B_{w 1}\right) \bar{B}_{1 c}+B_{w 1}\right]\left[\left(1-u_{w 1}\right) \iota\right.$

$\left.\left.B_{w 1}\right) B_{1 c}+B_{w 1}\right]\left[\left(1-\bar{u}_{w 1}\right) \bar{u}_{1 c}+\bar{u}_{w 1}\right]^{2}\left[\left(\bar{R}-\bar{y}_{0}\right) \eta+\bar{y}_{0}\right] d \eta+$

$$
\begin{aligned}
& +i_{0}\left(\bar{R}-\bar{y}_{0}\right) \int_{0}^{1}\left[\left(1-B_{w 1}\right)+\left(B_{w 1}-1\right) \bar{B}_{1 c}\right] . \\
& \cdot\left[\left(1-\bar{u}_{w 1}\right) \bar{u}_{2 c}+\bar{u}_{w 1}\right]^{2}\left[\left(\bar{R}-\bar{y}_{0}\right) \eta+\bar{y}_{0}\right] d \eta= \\
& =\frac{1}{\sqrt{1-\bar{p}}}\left\{\left(\bar{R}_{c}-\bar{y}_{0 c}\right) \int_{0}^{1}\left[\bar{B}_{1 c} \bar{u}_{1 c}^{2}+i_{0}\left(1-\bar{B}_{1 c}\right) \bar{u}_{2 c}^{2}\right]\right. \text {. } \\
& \left.\cdot\left[\left(\bar{R}_{c}-\bar{y}_{0 c}\right) \eta+\bar{y}_{0 c}\right] d \eta+\frac{\bar{y}_{0 c}^{2}}{2}-\bar{R}^{2} \frac{\bar{p}}{4}\right\} \\
& \frac{1}{2}\left[\left(1-\bar{u}_{w 1}\right)+\left(\bar{u}_{w 1}-1\right) \bar{u}_{1 c}^{*}\right] \frac{d}{d \zeta}\left[(1-\bar{p}) \bar{y}_{0}^{2}\right]+ \\
& +\frac{d}{d \zeta}(1-\bar{p})\left\{\left(\bar{R}-\bar{y}_{0}\right) \int_{0}^{\eta^{*}}\left[\left(1-B_{w 1}\right) \bar{B}_{1 c}+B_{w 1}\right] .\right. \\
& \cdot\left[\left(1-\bar{u}_{w 1}\right) \bar{u}_{1 c}+\bar{u}_{w 1}\right]^{2}\left[\left(\bar{R}-\bar{y}_{0}\right) \eta+\bar{y}_{0}\right] d \eta+ \\
& +i_{0}\left(\bar{R}-\bar{y}_{0}\right) \int_{0}^{\eta^{*}}\left[\left(1-B_{w 1}\right)+\left(B_{w 1}-1\right) \bar{B}_{1 c}\right] \text {. } \\
& \left.\cdot\left[\left(1-\bar{u}_{w 1}\right) \bar{u}_{2 c}+\bar{u}_{w 1}\right]^{2}\left[\left(\bar{R}-\bar{y}_{0}\right) \eta+\bar{y}_{0}\right] d \eta\right\}+ \\
& -\sqrt{1-\bar{p}}\left[\left(1-\bar{u}_{w 1}\right) \bar{u}_{1 c}^{*}+\bar{u}_{w 1}\right] \frac{d}{d \zeta}\left(\bar{R}-\bar{y}_{0}\right) \cdot \\
& \cdot \int_{0}^{\eta^{*}}\left[\left(1-B_{w 1}\right) \bar{B}_{1 c}+B_{w 1}\right]\left[\left(1-\bar{u}_{w 1}\right) \bar{u}_{1 c}+\bar{u}_{w 1}\right] . \\
& \cdot\left[\left(\bar{R}-\bar{y}_{0}\right) \eta+\bar{y}_{0}\right] \sqrt{1-\bar{p}} d \eta+ \\
& -\sqrt{1-\bar{p}} i_{0}\left[\left(1-\bar{u}_{w 1}\right) \bar{u}_{2 c}^{*}+\bar{u}_{w 1}\right] \frac{d}{d \zeta} \sqrt{1-\bar{p}}\left(\bar{R}-\bar{y}_{0}\right) . \\
& \cdot \int_{0}^{\eta^{*}}\left[\left(1-B_{w 1}\right)+\left(B_{w 1}-1\right) \bar{B}_{1 c}\right]\left[\left(1-\bar{u}_{w 1}\right) \bar{u}_{2 c}+\bar{u}_{w 1}\right] .
\end{aligned}
$$

$$
\begin{gathered}
\cdot\left[\left(\bar{R}-\bar{y}_{0}\right) \eta+\bar{y}_{0}\right] d \eta=(1-\bar{p})\left[\left(\bar{R}-\bar{y}_{0}\right) \eta^{*}+\bar{y}_{0}\right] \cdot \\
\cdot\left(1-\bar{u}_{w 1}\right)^{2}\left\{\left[\left(1-B_{w 1}\right) \bar{B}_{1 c}^{*}+B_{w 1}\right]\left(\frac{d \bar{u}_{1 c}}{d \zeta}\right)^{*}+\right. \\
\left.+i_{0} \kappa_{21}\left[\left(1-B_{w 1}\right)+\left(B_{w 1}-1\right) \bar{B}_{1 c}^{*}\right]\left(\frac{d \bar{u}_{2 c}}{d \zeta}\right)^{*}\right\}+ \\
-0.25\left[\left(\bar{R}-\bar{y}_{0}\right) \eta+\bar{y}_{0}\right]^{2} \frac{d \bar{p}}{d \zeta}
\end{gathered}
$$

\subsubsection{The Boundary Conditions}

Boundary conditions for the equation array (17) are stated as follows:

$$
\begin{gathered}
\zeta=0, y=y_{0 c}, h=h_{c}, B_{w l}=0, \bar{B}_{1 c}=B_{1 c}, \\
\bar{R}=\bar{R}_{c}, \bar{u}_{w 1}=0, \bar{p}=0 .
\end{gathered}
$$

Here are: $\zeta=\kappa_{1} \bar{x}, \kappa_{21}=\kappa_{2} / \kappa_{1}$.

The conditions (18) mean that at the beginning of the confined jet its radius afterwards is constant and equal to the radius of the channel. The function $h$ is assumed constant in the channel, Function-indicator of the first phase equal to zero (absence of the first phase at the external boundary of the mixing layer). Velocity at the wall is zero due to nonslip at it.

\subsubsection{The Momentum Equation Added by $x \geq x_{i}$}

By $x \geq x_{i}$, when the initial part of a jet ends, also the momentum equation at the axis is added:

$$
\begin{gathered}
2 \sqrt{1-\bar{p}} \bar{R}^{2} \int_{0}^{1}\left[\left(B_{m 1}-B_{w 1}\right) \bar{B}_{1 i}+B_{w 1}\right] . \\
\cdot\left[\left(1-\bar{u}_{w 1}\right) \bar{u}_{1 c}+\bar{u}_{w 1}\right] \eta d \eta=1, \\
\sqrt{1-\bar{p}} \bar{R}^{2} \int_{0}^{1}\left[\left(B_{w 1}-B_{m 1}\right) \bar{B}_{1 i}+1-B_{w 1}\right] . \\
\cdot\left[\left(1-\bar{u}_{w 1}\right) \bar{u}_{2 c}+\bar{u}_{w 1}\right] \eta d \eta=\bar{R}_{i}^{2} \int_{0}^{1}\left(1-B_{1 i}\right) \bar{u}_{2 c} \eta d \eta, \\
\int_{0}^{1}\left[\left(B_{m 1}-B_{w 1}\right) \bar{B}_{1 i}+B_{w 1}\right]\left[\left(1-\bar{u}_{w 1}\right) \bar{u}_{1 c}+\bar{u}_{w 1}\right]^{2} \eta d \eta+ \\
+i_{0} \int_{0}^{1}\left[\left(1-B_{w 1}\right)+\left(B_{w 1}-B_{m 1}\right) \bar{B}_{1 i}\right] \cdot \\
\cdot\left[\left(1-\bar{u}_{w 1}\right) \bar{u}_{2 c}+\bar{u}_{w 1}\right]^{2} \eta d \eta=\left\{\int _ { 0 } ^ { 1 } \left[B_{w 1 i}+\right.\right. \\
\left.+\left(1-B_{w 1 i}\right) \bar{B}_{1 i}\right]\left[\left(1-\bar{u}_{w 1 i}\right) \bar{u}_{1 c}+\bar{u}_{w 1 i}\right]^{2} \eta d \eta+
\end{gathered}
$$




$$
\begin{aligned}
& +i_{0} \int_{0}^{1}\left[\left(1-\bar{u}_{w 1 i}\right) \bar{u}_{2 c}+\bar{u}_{w 1 i}\right]^{2}\left[\left(1-B_{w 1 i}\right)+\right. \\
& \left.\left.+\left(B_{w 1 i}-1\right) \bar{B}_{1 i}\right] \eta d \eta=\frac{\bar{p}_{i}-\bar{p}}{4}\right\} \frac{\bar{R}_{i}^{2}}{(1-\bar{p}) \bar{R}^{2}}, \\
& \frac{d}{d \zeta} \bar{R}^{2}(1-\bar{p})\left\{-\bar{y}_{0} \int_{0}^{\eta^{*}}\left[\left(B_{m 1}-B_{w 1}\right) \bar{B}_{1 i}+B_{w 1}\right]\right. \text {. } \\
& \cdot\left[\left(1-\bar{u}_{w 1}\right) \bar{u}_{1 c}+\bar{u}_{w 1}\right]^{2} \eta d \eta+i_{0} \int_{0}^{\eta^{*}}\left[\left(1-B_{w 1}\right)+\right. \\
& \left.\left.+\left(B_{w 1}-B_{m 1}\right) \bar{B}_{1 i}\right]\left[\left(1-\bar{u}_{w 1}\right) \bar{u}_{2 c}+\bar{u}_{w 1}\right]^{2} \eta d \eta\right\}+ \\
& -\sqrt{1-\bar{p}}\left[\left(1-\bar{u}_{w 1}\right) \bar{u}_{1 c}^{*}+\bar{u}_{w 1}\right] \frac{d}{d \zeta} \bar{R}^{2} \sqrt{1-\bar{p}} \int_{0}^{\eta^{*}}\left[B_{w 1}+\right. \\
& \left.+\left(B_{m 1}-B_{w 1}\right) \bar{B}_{1 i}\right]\left[\left(1-\bar{u}_{w 1}\right) \bar{u}_{1 c}+\bar{u}_{w 1}\right] \eta d \eta+ \\
& -i_{0} \sqrt{1-\bar{p}}\left[\left(1-\bar{u}_{w 1}\right) \bar{u}_{2 c}^{*}+\bar{u}_{w 1}\right] \frac{d}{d \zeta} \sqrt{1-\bar{p}} \bar{R}^{2} \int_{0}^{\eta^{*}}[1+ \\
& \left.-B_{w 1}+\left(B_{m 1}-B_{w 1}\right) \bar{B}_{1 i}\right]\left[\left(1-\bar{u}_{w 1}\right) \bar{u}_{2 c}+\bar{u}_{w 1}\right] \eta d \eta= \\
& =(1-\bar{p}) \bar{R}^{2} \eta^{*}\left(1-\bar{u}_{w 1}\right)^{2}\left\{\left[\left(B_{m 1}-B_{w 1}\right) \bar{B}_{1 i}^{*}+B_{w 1}\right]\right. \text {. } \\
& \cdot\left(\frac{d \bar{u}_{1 c}}{d \eta} /\right)^{*}+i_{0} \kappa_{21}\left[\left(1-B_{w 1}\right)+\left(B_{w 1}-B_{m 1}\right) \bar{B}_{1 i}^{*}\right] \text {. } \\
& \left.\cdot\left(\frac{d \bar{u}_{2 c}}{d \eta}\right)^{*}\right\}-0.25\left(\eta^{*}\right)^{2} \bar{R}^{2} \frac{d \bar{p}}{d \zeta} \\
& \bar{u}_{m 1}\left[B_{m 1}+i_{0}\left(1-B_{m 1}\right)\right] \frac{d \bar{u}_{m 1}}{d \zeta}=2 \bar{u}_{m 1}^{2}\left(1-\bar{u}_{m 1}\right)^{2} . \\
& \cdot\left\lfloor B_{m 1}\left(\frac{d^{2} \bar{u}_{1 c}}{d \eta^{2}}\right)_{0}+i_{0} \kappa_{21}\left(\frac{d^{2} \bar{u}_{2 c}}{d \eta^{2}}\right)_{0}\left(1-B_{m 1}\right)\right\rfloor-\frac{1}{2} \frac{d \bar{p}}{d \zeta} \text {. }
\end{aligned}
$$

the immiscible liquids confined by the cylindrical channel.

In assumption of a slip on the wall of channel, the profiles of the main parameters in the form (14) are adopted with the following assignments:

$$
\begin{gathered}
u_{m i}=\bar{u}_{m i} u_{m i c}, u_{w i}=\bar{u}_{w i} u_{m i c}, B_{m 1}=B_{m 1 c} \bar{B}_{m 1}, \\
B_{w 1}=B_{m 1 c} \bar{B}_{w 1}, B_{1 c}=B_{m 1 c} \bar{B}_{1 c} .
\end{gathered}
$$

The pressure in a free jet is $p_{0}$.

\subsection{The Jet Confined at its Ground Part}

Then assuming similar to a free jet $u_{m 2}=u_{m l} S_{0}$, $u_{w 2}=u_{w 1} S_{0}$, the next dimensionless equation array for the jet confined at its ground part yields:

$$
\begin{aligned}
& \bar{B}_{m 1}\left[\left(\bar{u}_{m 1}-\bar{u}_{w 1}\right) \int_{0}^{1} \bar{B}_{1 c} \bar{u}_{1 c} \eta d \eta+\bar{u}_{w 1} \int_{0}^{1} \bar{B}_{1 c} \eta d \eta\right]+ \\
& \bar{B}_{w 1}\left[\left(\bar{u}_{m 1}-\bar{u}_{w 1}\right)\left(\int_{0}^{1} \bar{u}_{1 c} \eta d \eta-\int_{0}^{1} \bar{B}_{1 c} \bar{u}_{1 c} \eta d \eta\right)+\right. \\
& \left.+\left(\frac{1}{2}-\int_{0}^{1} \bar{B}_{1 c} \eta d \eta\right) \bar{u}_{w 1}\right]=\int_{0}^{1} \bar{B}_{1 c} \bar{u}_{1 c} \eta d \eta, \\
& \bar{B}_{m 1}\left[\left(\bar{u}_{m 1}-\bar{u}_{w 1}\right) \int_{0}^{1} \bar{B}_{1 c} \bar{u}_{2 c} \eta d \eta+\bar{u}_{w 1} \int_{0}^{1} \bar{B}_{1 c} \eta d \eta\right]+ \\
& +\bar{B}_{w 1}\left[\left(\bar{u}_{m 1}-\bar{u}_{w 1}\right)\left(\int_{0}^{1} \bar{u}_{2 c} \eta d \eta-\int_{0}^{1} \bar{B}_{1 c} \bar{u}_{2 c} \eta d \eta\right)+\right. \\
& \left.+\left(0.5-\int_{0}^{1} \bar{B}_{1 c} \eta d \eta\right) \bar{u}_{w 1}\right]=\int_{0}^{1} \bar{B}_{1 c} \bar{u}_{2 c} \eta d \eta+ \\
& +\frac{1}{B_{m 1 c}}\left[\left(\bar{u}_{m 1}-\bar{u}_{w 1}-1\right) \int_{0}^{1} \bar{u}_{2 c} \eta d \eta+0.5 \bar{u}_{w 1}\right] \text {, } \\
& \bar{p}=4 B_{m 1 c}\left\{\int_{0}^{1} \bar{B}_{1 c} \bar{u}_{1 c}^{2} \eta d \eta+i_{0} \int_{0}^{1}\left(\frac{1}{B_{m 1 c}}-\bar{B}_{1 c}\right) \bar{u}_{2 c} \eta d \eta+\right. \\
& +\left(B_{m 1}-B_{w 1}\right)\left[\left(\bar{u}_{m 1}-\bar{u}_{w 1}\right)^{2} \int_{0}^{1} \bar{B}_{1 c}\left(i_{0} \bar{u}_{2 c}^{2}-\bar{u}_{1 c}^{2}\right) \eta d \eta+\right. \\
& +2 \bar{u}_{w 1}\left(\bar{u}_{m 1}-\bar{u}_{w 1}\right) \int_{0}^{1} \bar{B}_{1 c}\left(i_{0} \bar{u}_{2 c}^{2}-\bar{u}_{1 c}\right) \eta d \eta+\left(i_{0}-1\right) \bar{u}_{w 1}^{2} . \\
& \left.\cdot \int_{0}^{1} \bar{B}_{1 c} \eta d \eta\right]+\bar{B}_{w 1}\left[\left(\bar{u}_{m 1}-\bar{u}_{w 1}\right)^{2}\left(i_{0} \int_{0}^{1} \bar{u}_{2 c} \eta d \eta-\int_{0}^{1} \bar{u}_{1 c}^{2} \eta d \eta\right)+\right. \\
& \left.+2 \bar{u}_{w 1}\left(\bar{u}_{m 1}-\bar{u}_{w 1}\right)\left(i_{0} \int_{0}^{1} \bar{u}_{2 c} \eta d \eta-\int_{0}^{1} \bar{u}_{1 c} \eta d \eta\right)+0.5\left(i_{0}-1\right) \bar{u}_{w 1}^{2}\right]+ \\
& -\frac{i_{0}}{B_{m 1 c}}\left[\left(\bar{u}_{m 1}-\bar{u}_{w 1}\right)^{2} \int_{0}^{1} \bar{u}_{2 c}^{2} \eta d \eta+\right.
\end{aligned}
$$

1.3.3 Boundary Conditions by $x \geq x_{i}$

$$
\begin{gathered}
\zeta=0, B_{m l}=1, \quad B_{w l}=B_{w l i}, \quad \bar{u}_{w 1}=\bar{u}_{w 1 i}, \\
\bar{u}_{m 1}=\bar{u}_{c 1 i}, \quad \bar{p}=\bar{p}_{i} .
\end{gathered}
$$

where $\quad \bar{B}_{1 i}=\bar{B}_{1}\left(x_{i}\right), \quad \bar{B}_{w 1 i}=\bar{B}_{w 1}\left(x_{i}\right), \quad \bar{p}_{i}=\bar{p}\left(x_{i}\right)$, $\zeta=\kappa_{1}\left(x-x_{i}\right) / r_{0}, \bar{u}_{m 1}=u_{m 1} / u_{01}, \bar{u}_{c 1 i}=\sqrt{1-\bar{p}_{i}}$.

The derived systems (17)-(20) of the mixed type equations and boundary conditions represent the mathematical model for two-phase turbulent jet of 


$$
\begin{aligned}
& \left.\left.2 \bar{u}_{w 1}\left(\bar{u}_{m 1}-\bar{u}_{w 1}\right) \int_{0}^{1} \bar{u}_{2 c} \eta d \eta+\frac{\bar{u}_{w 1}^{2}}{2}\right]\right\}, \\
& \frac{d}{d \bar{x}}\left\{\int _ { 0 } ^ { \eta ^ { * } } [ ( \overline { B } _ { m 1 } - \overline { B } _ { w 1 } ) \overline { B } _ { 1 c } + \overline { B } _ { w 1 } ] \left[\left(\bar{u}_{m 1}-\bar{u}_{w 1}\right) \bar{u}_{1 c}+\right.\right. \\
& \left.+\bar{u}_{w 1}\right]^{2} \eta d \eta+i_{0} \int_{0}^{\eta_{0}^{*}}\left[\frac{1}{B_{m 1 c}}-\left(\bar{B}_{m 1}-\bar{B}_{w 1}\right) \bar{B}_{1 c}-\bar{B}_{w 1}\right] . \\
& \left.\cdot\left[\bar{u}_{w 1}+\left(\bar{u}_{m 1}-\bar{u}_{w 1}\right) \bar{u}_{2 c}\right]^{2} \eta d \eta\right\}-\left[\left(\bar{u}_{m 1}-\bar{u}_{w 1}\right) \bar{u}_{1 c}^{*}+\bar{u}_{w 1}\right] \\
& \frac{d}{d \bar{x}} \int_{0}^{\eta^{*}}\left[\left(\bar{B}_{m 1}-\bar{B}_{w 1}\right) \bar{B}_{1 c}+\bar{B}_{w 1}\right]\left[\left(\bar{u}_{m 1}-\bar{u}_{w 1}\right) \bar{u}_{1 c}+\bar{u}_{w 1}\right] \eta d \eta+ \\
& -i_{0}\left[\left(\bar{u}_{m 1}-\bar{u}_{w 1}\right) \bar{u}_{2 c}^{*}+\bar{u}_{w 1}\right] \frac{d}{d \bar{x}} \int_{0}^{\eta^{*}}\left[\bar{B}_{w 1}+\frac{1}{B_{m 1 c}}+\right. \\
& \left.+\left(\bar{B}_{w 1}-\bar{B}_{m 1}\right) \bar{B}_{1 c}\right\rfloor\left[\left(\bar{u}_{m 1}-\bar{u}_{w 1}\right) \bar{u}_{2 c}+\bar{u}_{w 1}\right\rfloor \eta d \eta= \\
& =\kappa_{1} \eta^{*}\left(\bar{u}_{m 1}-\bar{u}_{w 1}\right)^{2}\left\{[ ( \overline { B } _ { m 1 } - \overline { B } _ { w 1 } ) \overline { B } _ { 1 c } ^ { * } + \overline { B } _ { w 1 } ] \left[\left(\frac{d \bar{u}_{1 c}}{d \eta}\right)^{*}\right.\right. \\
& \left.\left.-i_{0} \kappa_{21}\left(\frac{d \bar{u}_{2 c}}{d \eta}\right)^{*}\right]+i_{0} \kappa_{21}\left(\frac{d \bar{u}_{2 c}}{d \eta}\right)^{*}\right\}-\frac{\left(\eta^{*}\right)^{2}}{4 B_{m 1 c}} \frac{d \bar{p}}{d \bar{x}}, \\
& {\left[\bar{B}_{m 1}+i_{0}\left(1-\bar{B}_{m 1}\right)\right] \bar{u}_{m 1} \frac{d \bar{u}_{m 1}}{d \bar{x}}=\frac{-1}{2 B_{m 1 c}} \frac{d \bar{p}}{d \bar{x}}+2 \kappa_{1}\left(\bar{u}_{m 1}+\right.} \\
& \left.-\bar{u}_{w 1}\right)^{2}\left\lfloor\bar{B}_{m 1}\left(\frac{d^{2} \bar{u}_{1 c}}{d \eta^{2}}\right)_{0}+i_{0} \kappa_{21}\left(1-\bar{B}_{m 1}\right)\left(\frac{d^{2} \bar{u}_{2 c}}{d \eta^{2}}\right)_{0}\right\rfloor \text {. }
\end{aligned}
$$

The boundary conditions are:

$$
\bar{x}=0, \bar{u}_{w 1}=0, \bar{B}_{w 1}=0, \bar{u}_{m 1}=1, \bar{B}_{m 1}=1 .
$$

The mathematical models developed for the confined jets have been implemented for computer simulation.

\section{Numerical Simulation of Turbulent Two-Phase Confined Jet}

Numerical solution of the boundary-value problem (21), (22) has been done for a wide range of the varying parameters. The results are given in Fig. 4.

From the algebraic subsystem of the equation array (21), the parameters $\bar{B}_{m 1}, \bar{B}_{w 1}, \bar{p}$ were expressed as functions of the velocity components at the axis and at the wall: $\bar{u}_{m 1}, \bar{u}_{w 1}$. Then the system of two ordinary differential equations for the functions $\bar{u}_{m 1}(\bar{x}), \bar{u}_{w 1}(\bar{x})$ was solved numerically.
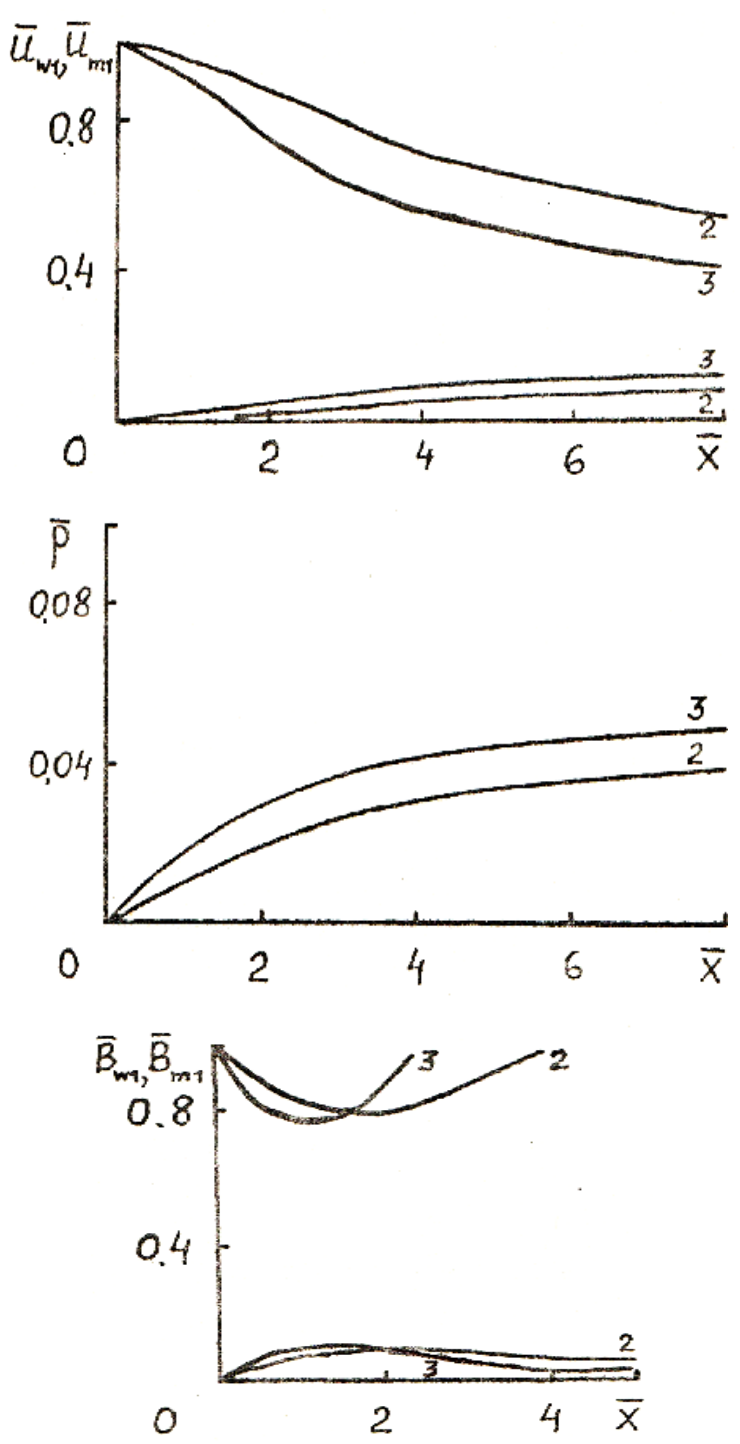

Fig. 4 Velocities, pressure and functionsindicators for the axis and wall of the channel:

$$
i_{0}=0.3, B_{m l c}=0.6, u_{m l c}=0.8, \kappa_{21}=3.6 \text {, }
$$

two variants: $2-\kappa_{1}=0.006,3-\kappa_{1}=0.008$.

\subsection{Analysis of Peculiarities of the Confined Two-Phase Jet}

Velocity of the confined jet at the axis of channel is slightly falling down (approximately 0.4-0.6) and growing at the wall (up to 0.08-0.12). Pressure in a channel is growing intensively at the entrance and slowly afterwards, nearly linearly. The most interesting is peculiarity of the functions-indicators at the axis and at the wall.

As clearly seen from the Fig. 4, the turbulent mixing in a first phase determines a length of the function variation with the longitudinal coordinate but not the function's character.

The higher is a turbulent mixing, the shorter is a distance of the varying process but the character and 
even the value is the same. The first phase at the axis is decreasing shortly at the entrance of the channel approximately $20 \%$, but then it recovers to the previous level $(100 \%$ first phase at the axis). Correspondingly, the first phase at the wall is growing on the same distance from 0 up to $10 \%$ and then falls down below $5 \%$.

This calculation was done for the case of lower density of a second phase. Thus, the heavier first phase at the beginning of the channel is distributed a little across the channel. But then it is nearly totally collected around the axis in the center of channel, which an interesting feature for the practice.

Numerical simulation revealed peculiarities different from the earlier considered free two-phase jet flow $[1-3,6,7]$. The functions $\bar{u}_{m 1}, \bar{u}_{w 1}$ have strong influence on the $\bar{B}_{m 1}, \bar{B}_{w 1}$, especially strong as concern to $\bar{B}_{w 1}$. This causes fast growing of the calculations' inaccuracy because the values $\bar{u}_{m 1}$, $\bar{u}_{w 1}$ by small errors in numerical solution lead to the increased errors in $\bar{B}_{m 1}, \bar{B}_{w 1}$, which are strongly inside the interval from 0 to 1 . Such peculiarities of the interconnection of the computed parameters cause serious impediments in computer simulations.

\subsection{The Equilibrium Two-Phase Flow Confined in Cyndrical Channel}

It is interesting to consider the equilibrium flow in a channel. As far as the inverse influence of $\bar{B}_{m 1}, \bar{B}_{w 1}$ to the values $\bar{u}_{m 1}, \bar{u}_{w 1}$ is small, we can write:

$$
\bar{x} \rightarrow \infty, \bar{B}_{m 1} \rightarrow \bar{B}_{w 1} \rightarrow \bar{B}_{1 \infty}, \bar{u}_{m 1} \rightarrow \bar{u}_{w 1} \rightarrow \bar{u}_{1 \infty} .
$$

The condition (11) is about total uniform distribution of the phases and their parameters in a cross-section of a chamber, which may be not achievable. But we can study this question. The following piecewise-linear approximations satisfy the following conditions:

$$
\begin{gathered}
\bar{B}_{m 1}=1+\left(\bar{B}_{1 \infty}-1\right) \sum_{k=1}^{m_{1}} b_{1 k} \bar{x}^{k}\left(1+\sum_{l=1}^{m_{1}} b_{2 l} \bar{x}^{l}\right)^{-1}, \\
\bar{B}_{w 1}=\bar{B}_{1 \infty} \sum_{j=1}^{m_{2}} t_{1 j} \bar{x}^{j}\left(1+\sum_{l=1}^{m_{2}} t_{2 i} \bar{x}^{i}\right)^{-1},
\end{gathered}
$$

where $b_{1 k}, b_{2 l}, t_{1 j}, t_{2 i}$ are constants computed from the equations (21). In general, their number is $2\left(m_{l}+\right.$ $\left.m_{2}\right)$. As far as the above task of finding the coefficients is very cumbersome already in a first approach, it was proposed accounting a weak influence of the $\bar{B}_{m 1}, \bar{B}_{w 1}$ on $\bar{u}_{m 1}, \bar{u}_{w 1}$, adopt for the functions $\bar{B}_{m 1}, \bar{B}_{w 1}$ the following simple approximations:

$$
\bar{B}_{m 1}=1+\left(\bar{B}_{1 \infty}-1\right) \frac{b_{1} \tilde{x}}{1+b_{2} \tilde{x}}, \quad \bar{B}_{w 1}=\bar{B}_{1 \infty} \frac{t_{1} \tilde{x}}{1+t_{2} \tilde{x}} .
$$

We stated the following conditions:

$$
\begin{gathered}
\tilde{x}=1, \bar{B}_{m 1}=\bar{B}_{w 1}=\bar{B}_{1 \infty}, \\
\tilde{x}=0.5, \quad \bar{B}_{m 1}=1.01 \bar{B}_{1 \infty}, \quad \bar{B}_{w 1}=0.99 \bar{B}_{1 \infty} ;
\end{gathered}
$$

where $\tilde{x}=\chi \bar{x}, \chi=\chi\left(i_{0}, x_{c}, \bar{x}\right)$ is some empirical function, e.g. $\chi=\chi\left(i_{0}, x_{c}\right)=$ const, in particular.

Then the following assignments were introduced:

$$
\begin{gathered}
\mathrm{AI} 5=\int_{0}^{1} \bar{B}_{1 c} \bar{u}_{1 c} \eta d \eta, \mathrm{AI} 8=\int_{0}^{1} \bar{u}_{1 c} \eta d \eta, \mathrm{AI} 11=\int_{0}^{1} \bar{u}_{1 c}^{2} \eta d \eta \\
\mathrm{AI} 14=\int_{0}^{1} \bar{B}_{1 c} \bar{u}_{1 c}^{2} \eta d \eta, \mathrm{AI} 15=\int_{0}^{1} \bar{B}_{1 c} \bar{u}_{2 c}^{2} \eta d \eta, \mathrm{AI} 16= \\
\int_{0}^{1} \bar{B}_{1 c} \bar{u}_{2 c} \eta d \eta, \mathrm{AEP}=\int_{0}^{1} \bar{u}_{2 c}^{2} \eta d \eta \\
\mathrm{BT}=\int_{0}^{1} \bar{u}_{2 c} \eta d \eta, \mathrm{PL}=\int_{0}^{1} \bar{B}_{1 c} \eta d \eta .
\end{gathered}
$$

Now from the first 3 equations (21), the following expressions yield for the introduced parameters:

$$
\begin{gathered}
\bar{u}_{1 \infty}=2\left[\mathrm{BT}+B_{m l c}(\mathrm{AI} 5-\mathrm{AI} 16)\right], \bar{B}_{1 \infty}=2 \mathrm{AI} 5 / \bar{u}_{1 \infty}, \bar{u}_{m 1} \\
=\left(1-\mathrm{F}_{2} \bar{u}_{w 1}\right), \bar{u}_{w 1}=\left(\mathrm{F}_{1} \mathrm{~F}_{5}-\mathrm{F}_{3}\right) /\left(\mathrm{F}_{1} \mathrm{~F}_{4}-\mathrm{F}_{2} \mathrm{~F}_{3}\right), \\
\bar{p}=4 B_{m 1 c}\left\{\mathrm{AI} 14+i_{0}\left(\mathrm{AEP} / B_{m 1 c}-\mathrm{AI} 15\right)+\right. \\
\left(\bar{B}_{m 1}-\bar{B}_{w 1}\right)\left[\left(\bar{u}_{m 1}-\bar{u}_{w 1}\right)^{2}\left(i_{0} \mathrm{AI} 15-\mathrm{AI} 14\right)+\right. \\
\left.+2\left(\bar{u}_{m 1}-\bar{u}_{w 1}\right) \bar{u}_{w 1}\left(i_{0} \mathrm{AI} 16-\mathrm{AI} 15\right)+\left(i_{0}-1\right) \mathrm{PL} \bar{u}_{w 1}^{2}\right] \\
+\bar{B}_{w 1}\left[\left(\bar{u}_{m 1}-\bar{u}_{w 1}\right)^{2}\left(i_{0} \mathrm{AEP}-\mathrm{AI} 11\right)+\right. \\
\left.+2 \bar{u}_{w 1}\left(\bar{u}_{m 1}-\bar{u}_{w 1}\right)\left(i_{0} \mathrm{BT}-\mathrm{AI} 8\right)+0.5\left(i_{0}-1\right) \bar{u}_{w 1}^{2}\right]+ \\
\left.-\frac{i_{0}}{B_{m 1 c}}\left[\frac{\bar{u}_{w 1}^{2}}{2}+\left(\bar{u}_{m 1}-\bar{u}_{w 1}\right)^{2} \mathrm{AEP}+2 \bar{u}_{w 1}\left(\bar{u}_{m 1}-\bar{u}_{w 1}\right) \mathrm{BT}\right]\right\},
\end{gathered}
$$

where are:

$$
\mathrm{F}_{5}=\mathrm{AI} 16-\mathrm{BT} / B_{m 1 c}, \mathrm{~F}_{4}=\bar{B}_{m 1}(\mathrm{PL}-\mathrm{AI} 16)+
$$




$$
\begin{gathered}
\bar{B}_{w 1}(0.5-\mathrm{PL}-\mathrm{BT}+\mathrm{AI} 16)+(\mathrm{BT}-0.5) / B_{m 1 c}, \\
\mathrm{~F}_{3}=\bar{B}_{m 1} \mathrm{AI} 16+\bar{B}_{w 1}(\mathrm{BT}-\mathrm{AI} 16)-\mathrm{BT} B_{m 1 c}, \\
\mathrm{~F}_{1}=\bar{B}_{m 1}+\bar{B}_{w 1}(\mathrm{AI} 8 / \mathrm{AI} 5-1), \mathrm{F}_{2}=\bar{B}_{m 1} . \\
\cdot(\mathrm{PL} / \mathrm{AI} 5-1)+\bar{B}_{w 1}[1+(0.5-\mathrm{PL}-\mathrm{AI} 8) / \mathrm{AI} 5] .
\end{gathered}
$$

\subsection{Empirical Constants in the Solution}

The obtained numerical solution of the boundary problem (21), (22) depends only on one empirical constant or function $\chi$, which is multiplayer in the variable $\tilde{x}=\chi^{\bar{x}}$ and does not depend on the constants of turbulent mixing in the phases $\kappa_{i}$. Therefore, it does not require introduction of any hypotheses concerning the turbulent shear stress.

The correlation of $\chi$ with empirical constants of the turbulent mixing $\kappa_{i}$ is easily computed from the solution of the two last equations (21).

\subsection{Parameters of Totally Stabilized Flow}

The parameters of the totally stabilized flow of confined two-phase turbulent jet of two immiscible liquids (e.g. $\bar{B}_{1 \infty}$ and the others) computed from the first three equations (21) are shown in Figs 5-7. Constants $t_{i}, b_{i}$ determined from substitution of (25) into (26), are given in the Table 1.

The three cases $i_{0}=0.3, B_{m l c}=0.6, u_{m l c}=0.8$, were analyzed as previously. Dimensionless velocity of the totally stabilized flow independently of $i_{0}$ and other parameters is close to 0.2 , while the functionindicator of the phase is substantially depending on

\begin{tabular}{|c|c|c|c|c|c|c|}
\hline$i_{0}$ & \multicolumn{2}{|r|}{0,3} & \multicolumn{2}{|r|}{, \pm 0} & \multicolumn{2}{|r|}{8,0} \\
\hline$B_{m 1 c}$ & 1,0 & $0,8 \quad 0,6$ & $I, 0$ & $0,8 \quad 0,6$ & $I, 0$ & $0,8 \quad 0,6$ \\
\hline$u_{m 1 c}$ & $I, 0$ & $0,920,8$ & $I, 0$ & $0,840,66$ & $I, 0$ & $0,530,20$ \\
\hline$R$ & 3,48 & 4, II 5,0 & 2,75 & $3,354,38$ & 2,00 & $3,18 \quad 5,0$ \\
\hline $\bar{u}_{1 \infty}$ & & $0,2 I$ & & 0,22 & 0,27 & $0,250,24$ \\
\hline$B_{100}$ & $D_{3} 3$ & $0,390,4$ & 0,58 & $0,60 \quad 0,6 I$ & 0,87 & $0,9 I I, 0$ \\
\hline $\bar{\rho}_{\infty}$ & 0, II & $0,124,14$ & 0,16 & $0,160,15$ & 0,19 & $0,340,8$ \\
\hline$b_{1}$ & 156 & $I 53 \quad I 50$ & 70 & $60 \quad 62$ & I4 & 8,50 \\
\hline$b_{2}$ & I55 & IF $I 49$ & 69 & क $6 \mathrm{I}$ & I3 & 7,50 \\
\hline$t_{1}$ & & 99 & & 99 & & 99 \\
\hline$t_{2}$ & & 98 & & 98 & & 38 \\
\hline
\end{tabular}
the parameter $i_{0}$ (density ratio multiplied by square of the slip ratio for the phases).

Table 1. Parameters of the stabilized confined jet

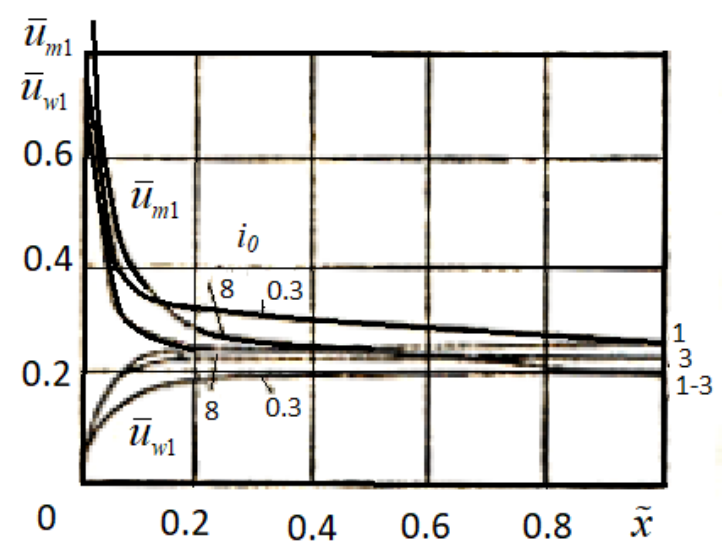

Fig. 5 Jet flow velocity in channel at axis $\bar{u}_{m 1}$ and at wall $\bar{u}_{w 1}$ against axial coordinate $\tilde{x}=\chi \bar{x}$ for $i_{0}=0.3$ and $i_{0}=8.0: 1-B_{m l c}=1.0,2-B_{m l c}=0.8,3-B_{m l c}=0.6$

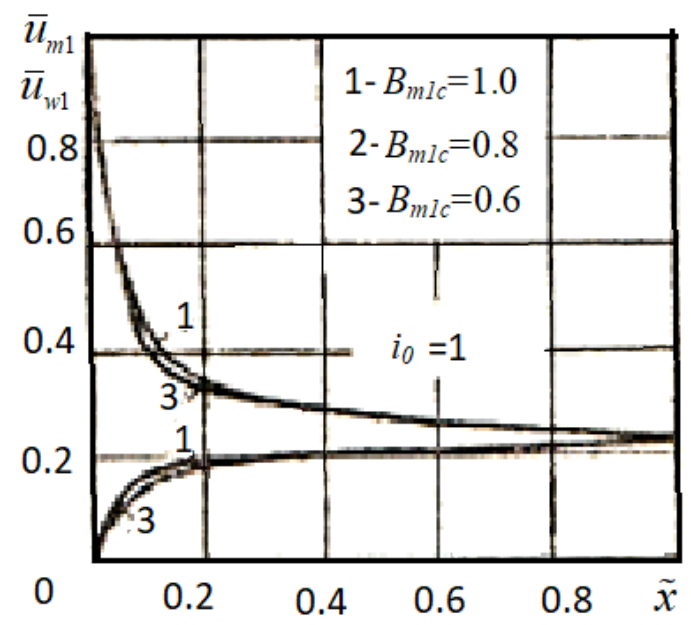

Fig. 6 Velocity of jet flow in channel at axis $\bar{u}_{m 1}$ and at wall $\bar{u}_{w 1}$ against axial coordinate $\tilde{x}=\chi \bar{x}$ for $i_{0}=1.0: 1-B_{m l c}=1.0,2-B_{m l c}=0.8,3-B_{m l c}=0.6$

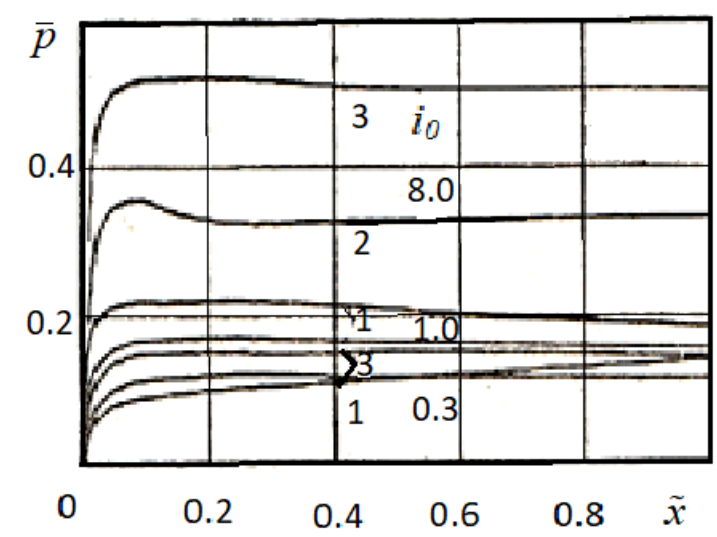

Fig. 7 Pressure of a jet flow in the cylindrical channel against axial coordinate for $i_{0}=0.3,1.0,8.0$ and: $1-B_{m l c}=1.0,2-B_{m l c}=0.8,3-B_{m l c}=0.6$

The velocities of flow and pressure are presented in Figs 5-7 against axis of the channel. It is clearly observed that the most intensive mixing is 
performed in the region by $\tilde{x} \leq 0.1$. Afterwards it is going slower and slower, especially concerning the pressure. Maximum of the recoverable pressure by a high density ratio allows deciding, in a frame of the model, in a first approach, about the rational length of the stabilizing channel.

Dimensionless pressure depends a little on a density ratio, which abruptly increases only by big density ratio and a small velocity of the jet's entrainment into a channel. The last case may evidence a possibility for the jamming a jet in a confining channel.

\section{Validation of the results obtained}

The results obtained by numerical solution of the boundary problem (21), (22) and its approximate solution was done with the known experimental data. By two-phase flows such data are absent; therefore, a comparison of the experiments by a turbulent mixing in the jet devices working on homogeneous flows of incompressible liquids is presented in Fig. 8:

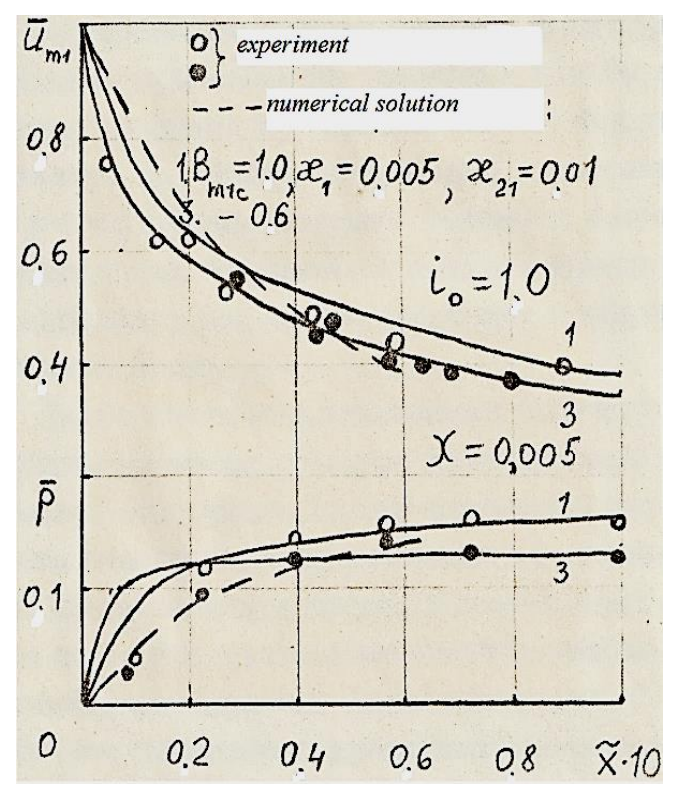

Fig. 6 Comparison of the solutions obtained against experimental data

For $\chi=0.005$, the correspondence of the results obtained with experimental data [9] is quite good. And it might be even slightly improved with choosing the function $\chi(\bar{x})$ for the best correlation in each specific case.

The method was successfully applied to modeling and simulation of the complex two-phase flows for the corium cooling during severe accidents at NPP [7] and to calculation of the parameters for the new jet type machines for steel melting [10].

\section{Short review of recent papers}

As far as this is the third paper in our series on the heterogeneous jets of mutually immiscible liquids, which we did not find analogues in the literature, we have to conclude with a short review of recent papers, which may be of interest to some partial phenomena considered by us and to further study.

The detailed measurements in confined coaxial turbulent jets were performed in [11] with a laser Doppler anemometer, especially in the initial mixing region, in order to study the physical phenomena and the existence of organized flow structures. The performance of the two-equation turbulence model was tested for selected previous experimental data.

The induced flow in a long cylinder by the axial round turbulent jet was investigated experimentally with applications to crude oil storage [12]. It was found that the flow does not reach a true steady state but vacillates periodically. Digital video recordings and particle image velocimetry were used, and the frequency of jet switching, jet stopping distance, turbulence characteristics, and the influence of endwall boundary conditions were inferred.

A systematic search for combined axial and helical forcing of a round jet that maximize mixing was described in [13], which concentrated on optimization of jets at higher Reynolds numbers using large eddy simulation.

The paper [15] presented study of the stochastic Lagrangian agglomeration model applied in the classical Euler-Lagrange approach. The main focus was on a modelling of the turbulent particle transport and particle collision and agglomeration. The dynamic equation of the individual particle motion was solved in the Lagrangian approach, while the particulate phase properties were given by the ensemble average in a number of particles.

Then the paper [16] identifies the mechanisms in the wall region and particle segregation in the viscous sublayer in the regions with streamwise fluid velocity lower than the mean ones, which is of paramount importance for many technological and environmental problems. The results from a direct numerical simulation of the passive transport of solid particles by a fully developed turbulent channel flow with a Reynolds number of 180 based on the friction velocity and the channel half-width has been presented in [17].

The mathematical model for the phenomenon of preferential concentration of inertial particles in a 
turbulent field was done in [18]. Various scaling limits of interest have been studied together with the effect of particle collisions. The effect of collisions was estimated negligible but in some cases particles may decrease diffusion.

The effect of spatial correlation between the particle velocities was investigated in two-phase turbulent flows [19] using the statistical model built on the joint probability distribution of the fluid and particles. Some singularities of utilization of LDA for study a structure of the gas-solid particles heterogeneous flows were considered [20], where revealed the behaviours of particles moving in a gas flow and their effect on the parameters of the carrier flow.

\section{Conclusion}

The results obtained may be useful in a number of chemical technology and other engineering fields, where the jet hydraulic machines are applied. Mathematical model developed for the free and confined jets of two-phase flows and the approximate correlations proposed from analysis are available for implementation into the research and engineering calculations.

Account of the phase spatial and temporal distribution in the mixing multiphase flows is important for deep understanding and optimization of the mixing processes. The model and experimental technique for the investigation of the multiphase turbulent jet of mutually immiscible liquids (metal melt-slag, oil-water, etc.), when two or more liquids in a mixed flow have their own dynamically changing interfaces, is unique and may be of interest in the mentioned cases.

The application of the method has some limitations in touch with approximations of the function-indicator and numerical solution of the boundary problems, which were detail investigated and the advices to fight them were developed.

\section{Acknowledgements}

The author devotes this paper in a series with earlier published two papers $[6,7]$ to the blessed memory of Prof. Alfred I. Nakorchevskii, who created this original method and experimental technique and was the first his mentor in scientific work. And the author wishes to acknowledge Prof. Torsten H. Fransson for the possibility to work at the Dept of Energy Technology, Royal Institute of Technology (KTH), during many years since 2001.

\section{References:}

[1] Nakorchevskii A.I. Heterogeneous turbulent jets. Kyiv: Naukova Dumka, 1980, 142 p.

[2] Nakorchevskii A.I., Kazachkov I.V. Calculation of heterogeneous turbulent jet. In: Automation Systems of continuous technological processes, Inst. of Cybernetics of NASU, 1979, P. 68-79.

[3] Kazachkov I.V. Approaches for mathematical modeling and experimental study of the turbulent flows of mutually immiscible liquids (oil-water)// Int. J. Petroleum and Petrochemical Engineering (IJPPE).- V. 4, N 1, 2018, P. 1-12.

[4] Nakorchevskii A.I., Basok B.I. Hydrodynamics and heat transfer in heterogeneous systems and devices of pulsating type. Kyiv: Naukova Dumka, 2001, 348 p.

[5] Kazachkov I.V. Mathematical modeling of heterogeneous turbulent jets in cylindrical chamber// Soviet automatic control, 1980, vol.13, jan.-feb., p. 1-6.

[6] Kazachkov Ivan V. Mathematical Modeling of the Mixing and Heat Transfer in Turbulent Two-Phase Jets of Mutually Immiscible Liquids// WSEAS Transactions on Heat and Mass Transfer, 2020, 15 (16), p. 117-129.

[7] Kazachkov Ivan V. Numerical Simulation of the Turbulent Two-Phase Jet// Engineering World, 2020, vol. 2, p. 107-119.

[8] Yakovlevskii O.V. The Mixing of Jets in a Channel with Variable Cross Section// Izvestiya Akademii NaukSSSR, OTN, Mekhanika i Mashinostroyeniye.- 1962, No. 1, p. 66-72 (English translation FTD-TT-62- 1571).

[9] Nakorchevskii A.I. Basic regularities of the stabilization of flows in jet devices. -In book: Hydraulics and hydrotechnics.- Kiev: Tekhnika.- 1966, №4, p. 127-145.

[10] Nakorchevskii A.I. Reactor for continuous refinement of metal/ Patent USSR №492554.Bulletin.- 1975.- №43.

[11] Khodadadi J.M. and Vlachost N.S. Experimental and Numerical Study of Confined Coaxial Turbulent Jets// AIAA Journal, 1989, Vol. 27, No. 5, p. 532-541.

[12] Voropayev S.I., Sanchez X., Nath C., Webb S., and Fernando H.J.S. Evolution of a confined turbulent jet in a long cylindrical cavity: Homogeneous fluids// Physics of Fluids, 2011, 23, 115106, p. 1-11.

[13] Hilgers A. Control and optimization of turbulent jet mixing/ Center for Turbulence Research: Annual Research Briefs, 2000, p. 4554. 
[14] Dreitser, G. A., Some problems of development of highly efficient tubular heat exchangers, Proc. V Minsk Int. Forum of Heat and Mass Transfer, Minsk, 2004, vol. 2, p. 288-289.

[15] Ho C.A. and Sommerfeld M. Modeling of micro-particle agglomeration in turbulent flows// Chem. Eng. Sci., 2002, vol. 57, p. 30733084.

[16] Marchioli C. and Soldati A. Mechanisms for particle transfer and segregation in a turbulent boundary layer// J. Fluid. Mech., 2002, vol. 468, p. 283-315.

[17] Rouson D.W.I. and Eaton J.K. On the preferential concentration of solid particles in turbulent channel flow// J. Fluid Mech., 2001, vol. 428, p. 149-169.

[18] Sigurgeirsson H. and Stuart A.M. A model for preferential concentration// Phys. Fluids, 2002, vol. 14, no. 12, p. 4352-4361.

[19] Simonin O., Fevrier P., and Lavieville J., On the spatial distribution of heavy particle velocities in turbulent flow: from continuous fi eld to particulate chaos// J. Turbulence, 2002, vol. 3 (040), p. 1-18.

[20] Varaksin A.Yu. and Polyakov A.F. Some problems associated with experimental investigation of the structure of heterogeneous flows// High Temp., 2000b, vol. 38, no. 4, p. 621-628.

\section{Creative Commons Attribution License 4.0 (Attribution 4.0 International, CC BY 4.0)}

This article is published under the terms of the Creative Commons Attribution License 4.0

https://creativecommons.org/licenses/by/4.0/deed.en US 